\title{
Prognostic value of reported chest pain for cardiovascular risk stratification in primary care
}

\author{
David M Leistner ${ }^{1}$, Jens Klotsche ${ }^{2}$, Sylvia Palm ${ }^{1}$, Lars Pieper $^{2}$, Günter K Stalla ${ }^{3}$, Hendrik \\ Lehnert ${ }^{4}$, Sigmund Silber ${ }^{5}$, Winfried März ${ }^{6}$, Hans-Ulrich Wittchen ${ }^{2}$ and Andreas M Zeiher ${ }^{1}$ \\ (for the DETECT Study Group)
}

\author{
1 Department of Internal Medicine III, Cardiology, Goethe-University Frankfurt, Germany \\ 2 Institute for Clinical Psychology and Psychotherapy, Technische Universität Dresden, Germany \\ 3 Max Planck Institute of Psychiatry, Munich, Germany \\ 4 Department of Medicine I, University of Schleswig-Holstein, Campus Lübeck, Germany \\ 5 Cardiology Practice and Hospital, Munich, Germany \\ 6 Synlab Center of Laboratory Diagnostics, Heidelberg, Germany
}

Corresponding author: Andreas M Zeiher, Department of Medicine III, Cardiology and Molecular Cardiology, Goethe-University of Frankfurt, Theodor-Stern-Kai. 7, D-60590 Frankfurt/Main, Germany. Email:

Zeiher@em.uni-frankfurt.de

\begin{abstract}
Background: The prognostic significance of chest pain is well established in patients with coronary artery disease, but still ill defined in primary prevention. Therefore, the aim of our analysis was to assess the prognostic value of different forms of chest pain in a large cohort of primary care subjects under the conditions of contemporary modalities of care in primary prevention, including measurement of serum levels of the biomarker NT-pro-BNP. Design: We carried out a post-hoc analysis of the prospective DETECT cohort study. Methods: In a total of 5570 unselected subjects, free of coronary artery disease, within the 55,518 participants of the cross-sectional DETECT study, we assessed chest pain history by a comprehensive questionnaire and measured serum NT-pro-BNP levels. Three types of chest pain, which were any chest pain, exertional chest pain and classical angina, were defined. Major adverse cardiovascular events (MACEs=cardiovascular death, myocardial infarction, coronary revascularization procedures) were assessed during a 5-year follow-up period. Results: During follow-up, 109 subjects experienced a MACE. All types of reported chest pain were associated with an approximately three-fold increased risk for the occurrence of incident MACEs, even after adjusting for cardiovascular risk factors. Any form of reported chest pain had a similar predictive value for MACEs as a one-time measurement of NT-proBNP. However, adding a single measurement of NT-pro-BNP and the information on chest pain resulted in reclassification of approximately $40 \%$ of subjects, when compared with risk prediction based on established cardiovascular risk factors.

Conclusions: In primary prevention, self-reported chest pain and a single measurement of NTpro-BNP substantially improve cardiovascular risk prediction and allow for risk reclassification of approximately $40 \%$ of the subjects compared with assessing classical cardiovascular risk factors alone.
\end{abstract}


Keywords: Self-reported chest pain, NT-pro-BNP, risk prediction, primary prevention

\section{Introduction}

Coronary artery disease (CAD) is one of the most prevalent diseases and remains the leading cause of death. ${ }^{1}$ Therefore, the identification of subjects at risk to develop CAD is a major goal in primary prevention.

In most cases, angina pectoris is a typical symptom of the initial presentation of CAD. ${ }^{2}$ Although the significance of angina is well known in patients with a history of CAD and it is the basis for diagnostic and therapeutic decisions in clinical practice, ${ }^{3-6}$ the prognostic value of angina as a predictor of future cardiovascular events is still ill defined in primary prevention. As the perception of angina is highly variable, especially for the elderly, women and diabetics, ${ }^{7-9}$ its diagnostic specificity and sensitivity varies between subgroups of patients. ${ }^{10,11}$

Therefore, the aim of our study was to analyse the prognostic value of different types of reported chest pain in a large cohort of unselected primary care subjects under the conditions of contemporary modalities in primary prevention including measurement of the biomarker $\mathrm{N}$-terminal pro-brain natriuretic peptide (NT-pro-BNP), which recently emerged as a valuable risk predictor of cardiovascular events in the general population. ${ }^{12,13}$

\section{Materials and methods}

The 'Diabetes Cardiovascular Risk Evaluation Targets and Essential Data for Commitment of Treatment' (DETECT) trial is a large multistage prospective longitudinal study. In brief, in the DETECT study, patients were recruited in a total of 3188 general practitioners (GPs) offices evenly distributed throughout Germany at two half-day time points. In detail, during the morning working hours (08:00-12:00) on 16 and 18 September 2003, all consecutive subjects visiting their GP for diagnostic or therapeutic counselling were recruited into the main DETECT study. ${ }^{14}$ In total, 55,518 subjects were included into the main DETECT study addressing the demographic and cardiovascular risk profile of primary care subjects in Germany. Thus, the recruitment of all consecutive subjects visiting their primary care physician at two predefined half-days indeed represents a cross-sectional snapshot of subjects seeking medical advice with their GP throughout Germany. Of the 3188 GPs, 1000 were randomly selected to additionally obtain blood samples for standardized laboratory screening in 5-10 randomly selected subjects out of the consecutive subjects visiting the GP office at the predefined half-day recruitment period. ${ }^{14}$ This cohort, comprising a total of 7519 subjects, was then followed-up for 5 years by their treating GP in order to document incident adverse clinical events. Importantly, the cohort of subjects with blood samples did not differ from the main 55,518 subjects of the DETECT cohort with respect to demographic and cardiovascular risk profiles, thus ascertaining recruitment of a subgroup of subjects representative for the entire study cohort, reflecting a truly unselected population. ${ }^{14}$ For the present analysis, subjects with a history of cardiovascular disease were excluded, resulting in a study cohort of 5570 subjects, in whom clinical chemistry could be performed from blood samples obtained at inclusion into the study and correlated with 5-year clinical outcome with respect to incident adverse cardiovascular events. ${ }^{14}$ Supplementary Table 1 illustrates the flowchart of recruitment of subjects into the present study. 


\section{Baseline examinations}

Subjects completed a self-administered questionnaire, which was used to assess demographic data, smoking history, family history of coronary artery disease, information on duration and severity of cardiovascular risk factors and existing medical as well as non-medical treatment. The questionnaire included multiple-choice questions on occurrence, location and characteristics of chest pain in order to derive three definitions of chest pain (Supplementary Table 2): (1) the standardized and validated 'Rose angina', ${ }^{15}$ which is still used by the World Health Organization (WHO) for the definition of angina and was already used in other studies, ${ }^{16}$ (2) the clinically most frequently used 'exertional chest pain' and 'any chest pain' as well as a simple classification of chest pain proposed by the National Institute for Health and Clinical Excellence (NICE) guidelines ${ }^{17}$ on the management of chest pain (typical angina, atypical angina and non-anginal chest pain).

Venous blood samples were immediately frozen after collection until the time of the analysis. Concentrations of plasma NT-pro-BNP was determined with a sandwich immunoassay on an ELECSYS2010 analyser (Roche diagnostics); ${ }^{18}$ Troponin T (TnT) was measured by the Elecsys 2010, third-generation assay (F. Hoffmann-La Roche Diagnostics, Basel, Switzerland).

Established cardiovascular risk factors were defined as follows: hypertension was defined as a systolic blood pressure $\geq 140 \mathrm{mmHg}$, a diastolic blood pressure $\geq 90 \mathrm{mmHg}$ or treatment with antihypertensive medication. ${ }^{19}$ National Cholesterol Education Program (NCEP) guidelines ${ }^{20}$ were applied for the diagnosis of dyslipidemia. Diabetes mellitus (DM) was defined by the use of antihyperglycaemic medication or by a fasting plasma glucose level $\geq 126 \mathrm{mg} / \mathrm{dl}$ (7.0 $\mathrm{mmol} / \mathrm{l}){ }^{21}$ Smoking was defined as current smoking, which includes both active daily nicotine abuse and occasional active smoking. An overall risk factor score was calculated using the Framingham risk score definitions. ${ }^{22}$

The DETECT survey received the approval of the Ethics Committee of the Carl Gustav Carus Medical Faculty at the Technical University of Dresden (AZ: EK149092003; Date: 16 September 2003) and was registered at ClinicalTrials.gov (NCT01076608).

\section{Endpoints}

At the 5-year follow-up in 2008, state of health and medical history over the follow-up period were ascertained. The following endpoints were documented: all cause mortality, mortality of cardiovascular cause, occurrence of a myocardial infarction, and manifestation of CAD as evidenced by the necessity for coronary revascularization by either coronary artery bypass graft $(\mathrm{CABG})$ surgery or percutaneous coronary intervention (PCI). Deaths and known causes of deaths were determined by a standardized assessment form by the primary care physician and/or by the institution in which the patient was previously treated. Cardiovascular death was defined as either sudden (out-of-hospital sudden death) or myocardial infarction- related requiring documentation of myocardial infarction by hospital records. Events were verified by reviewing the hospital charts of patients with non-fatal myocardial infarction or manifestation of CAD due to the necessity for revascularization procedures. Only the first event occurring during follow-up was used as an endpoint. In addition to the individual endpoints, a combined endpoint of major adverse cardiovascular events (MACEs) was used including death from cardiovascular causes, non-fatal myocardial infarction and necessity for coronary revascularization by CABG surgery or PCI. In addition we also analysed the data excluding 
revascularization as an endpoint given that reported angina might lead to invasive investigations associated with increased revascularization rates.

\section{Statistical analyses}

The association of baseline characteristics as well as the association of different definitions of chest pain with the outcome was investigated with the use of Cox proportional hazards regression. In addition to crude analysis, hazard ratios were adjusted for demographic factors (age and gender) as well as for established risk factors (arterial hypertension, diabetes mellitus, hyperlipidemia, obesity and smoking status). Estimates of the $\mathrm{C}$ statistic after Cox regression models (with 95\% confidence intervals), which is the nonparametric estimate of the area under the receiver operating characteristic (ROC) curve, for conventional cardiovascular risk factors and NT-pro-BNP with different types of chest pain were calculated to assess model discrimination. ${ }^{23}$

In addition, we evaluated the ability to use different questions on chest pain as a diagnostic tool, with and without NT-pro-BNP, to reclassify risk, according to previously suggested methods. ${ }^{24}$ Using multivariable risk models with the clinical covariates listed above, we calculated the net reclassification Improvement (NRI) and integrated discrimination improvement (IDI). ${ }^{24}$ Subjects were reclassified based on their predicted probabilities for the future event based on the addition of chest pain diagnosis and/or biomarker concentration. The number of subjects reclassified was assessed by the category free NRI. The difference between improvement in average sensitivity and potential increase in average 'one minus specificity' was calculated be the IDI. Moreover, quantile-quantile plots were constructed in order to delineate changes in estimated risk across the entire range of risk.

Results are presented as mean (standard deviation [SD]) for approximately normal distributed variables, median (interquartile range) for skewed variables and absolute and relative frequencies for categorical variables. Two-sided p-values of less than 0.05 were considered to indicate statistical significance. All statistical analyses were conducted with the use of STATA 11.

\section{Results}

The baseline characteristics of the study cohort and the association with MACEs are shown in Table 1. The mean age of participants at baseline was 55.6 years (SD 13.8 years; range 18-95 years). A total of 3465 participants $(62.2 \%)$ were women; 1900 participants $(34.1 \%)$ had arterial hypertension; 683 patients $(12.3 \%)$ suffered from diabetes mellitus. Pharmacological treatment consisted of angiotensinconverting enzyme (ACE) inhibitors/angiotensin receptor blockers (ARBs) $(23.5 \%)$, beta-blockers $(20.2 \%)$, calcium channel antagonists $(9.9 \%)$ or diuretics $(12.4 \%)$. None of the subjects had prevalent major cardiovascular disease at baseline. During the follow-up period of 5 years, 157 participants $(2.8 \%)$ died, 137 from noncardiac causes, and 20 from cardiovascular cause. A total of $109(2.0 \%)$ subjects experienced an incident MACE: in detail, 34 subjects suffered from non-fatal myocardial infarction and $1.5 \%(n=86)$ of the subjects underwent first revascularization procedures by PCI $(n=62)$ or by CABG $(n=24)$. As summarized in Table 1, the occurrence of a MACE was associated with the classical risk factors for $\mathrm{CAD}$, except smoking. Likewise, subjects suffering from incident MACEs during follow-up had significantly higher NT-pro-BNP serum levels as well as a 
higher incidence of elevated Troponin T (TnT) levels. However, it should be noted, that TnT levels were above the currently accepted threshold of $>0.01 \mathrm{pg} / \mathrm{dl}$ in only $0.4 \%$ of the entire study cohort, thus precluding its use as a clinically meaningful biomarker in primary prevention.

All types of reported chest pain, Rose angina, exertional chest pain and any chest pain, as well as all NICE angina classifications were associated with an approximately three-fold increased risk for the occurrence of MACE (Table 2). ${ }^{15,17}$ These associations remained unchanged after adjustment for age and gender or after additional adjustment for established cardiovascular risk factors. Most importantly, exclusion of revascularization from the combined MACE endpoint did not alter the results (Table 2), thus documenting that the observed associations between different types of reported chest pain and adverse outcome not exclusively driven by a higher likelihood of patients with chest pain undergo invasive investigations leading to an increased rate of revascularization. None of the types of chest pain increased the hazard ratios for all-cause mortality.

As illustrated in Supplementary Table 3, all definitions of reported chest pain were associated with a higher prevalence of established cardiovascular risk factors.

The C statistic for Cox regression models increased significantly for the prediction of major cardiovascular events, when all types of reported chest pain were separately incorporated into a model with the established cardiovascular risk factors (Table 3 and Supplementary Table 5), with Rose angina achieving only borderline significance. Moreover, when adding the combination of different types of chest pain and NT-pro-BNP to cardiovascular risk factors into the risk prediction model, a further significant increase of the $\mathrm{C}$ statistics for Cox regression models predicting MACEs was observed for all definitions of chest pain. Importantly, as summarized in Supplementary Table 6, the addition of NT-pro-BNP serum levels specifically increased the $\mathrm{C}$ statistics for all forms of NICE angina, whereas no added value was observed in subjects without angina.

Finally, we tested the usefulness of adding chest pain, alone and in combination with NT-proBNP serum levels, to conventional cardiovascular risk factors by assessing the number of participants reclassified and calculating the IDI on top of the Framingham 10-year risk prediction of a major cardiovascular event. As summarized in Table 4, integrated discrimination was significantly improved for major cardiovascular events, when all types of self-reported chest pain were incorporated into the risk model. Reclassification effect by adding the data on exertional chest pain was similar to measuring NT-pro-BNP serum levels (Table 4a).

Most importantly, however, combining data on self-reported chest pain and NT-pro-BNP serum levels resulted in a further significant improvement of integrated discrimination (Table 4b). In fact, approximately $40 \%$ of the subjects were reclassified either into a higher risk category for subjects with events or into a lower risk category in subjects without events. The incremental value of adding self-reported chest pain to conventional risk factors for improved risk prediction is also illustrated by the quantile-quantile plots shown in Figure 1, which demonstrate significant deviations from the line of identity indicating changes in estimated risk across the continuum of risk estimates for all types of chest pain.

\section{Discussion}


The results of the present study demonstrate that in a contemporary nationwide, crosssectional sample of primary care subjects, free of any evidence of CAD, all types of reported chest pain are associated with an approximately three-fold increase in risk for subsequent MACEs during a 5-year follow-up period. Importantly, the predictive power of reporting any form of chest pain was similar to the risk predictive value of measuring NT-pro-BNP serum levels. However, adding the information on chest pain, and a single measurement of NT-proBNP serum levels resulted in reclassification of approximately $40 \%$ of primary care subjects when compared with risk prediction based on established cardiovascular risk factors.

The prognostic significance of angina is well established in patients with established CAD. However, the few prognostic studies of angina in primary care provided inconclusive results. ${ }^{25}$ A recent study including 1785 subjects with a diagnosis of self-reported angina as their first manifestation of ischaemic heart disease demonstrated that men with angina are at significantly increased risk of suffering from major adverse cardiovascular events including death, acute myocardial infarction and revascularization procedures. ${ }^{26}$ The present approximately three-fold larger study not only extends these observations, but also reveals that various forms of reported angina provided similar incremental predictive information compared with measuring NT-pro-BNP serum levels. Thus, a simple question regarding the presence or absence of angina appears to provide predictive information as robust as NT-proBNP in this setting.

While recent years have witnessed great enthusiasm to use biomarkers as tools to enhance risk prediction in primary prevention populations, ${ }^{12,13,27,28}$ the incremental value of a variety of tested biomarkers to enhance risk prediction in primary prevention turned out to be rather small and resulted in only small, if any, improvement in discrimination and reclassification. ${ }^{28}$ Nevertheless, consistent throughout most of the studies, NT-pro-BNP serum levels demonstrated the greatest prognostic value in primary prevention subjects. ${ }^{12,13,27,29-31}$ The results of the present study confirm the utility of NT-pro-BNP as a biomarker for population screening. However, importantly, the incremental value of measuring NT-pro-BNP serum levels for cardiovascular risk prediction in primary prevention was restricted to subjects reporting angina.

Thus, adding NT-pro-BNP and all types of angina to established risk factors led to a further significant improvement in integrated discrimination. Thus, a simple question regarding any type of chest pain, combined with a single measurement of NT-pro-BNP serum levels results in reclassification of 5-year predicted risk of approximately $40 \%$ of subjects compared with the assessment of classical risk factors alone, providing indeed a time-efficient and feasible strategy for risk assessment in primary prevention (Figure 2).

\section{Limitations}

The definitions of self-reported angina included previously validated conventional categorization of chest pain, such as Rose angina ${ }^{15}$ as well as the simple NICE guidelines ${ }^{17}$ recommended classification, in order to allow for generalization of the results. Thus, we cannot rule out that more sophisticated questioning by the primary physician for more precise differentiation between the various forms of chest pain would have had an impact on the results. However, with respect to time constraints and immense workload in primary care physician practices, a time-efficient, standardized questionnaire of self-reported angina appears to be sufficient to effectively improve risk prediction, as demonstrated by the results of the present study. By design, the present study does not allow for the distinction between prevalent and incident cases of chest pain, as no data were obtained regarding the duration of 
chest pain symptoms. Diagnostic work-up and management of subjects with chronic chest pain (prevalent cases) may in fact have led to an underestimation of the observed association with MACEs. However, the results of our analyses did not change when excluding the necessity for revascularization as an endpoint. Thus, eliminating the 'self-fulfilling-prophecy' that subjects with incident chest pain will be more likely to undergo invasive coronary diagnostic work-up resulting in an increased incidence of revascularization procedures demonstrated, that the results of the present study are not exclusively driven by the lack of differentiation between incident and prevalent cases of angina. Furthermore in line with the general office hours of GPs in Germany, subjects were recruited during the morning hours. This may have a potential selection bias into the DETECT cohort.

Albeit rather small, the relative number of cardiovascular events in the present study with a 5year follow-up period is essentially identical to data from recently published European population-based studies in primary prevention, ${ }^{33}$ suggesting the usefulness of the DETECT cohort for cardiovascular risk estimation in a primary prevention population free of CAD.

In summary, the present study is the first to validate self-reported chest pain as a useful tool to enhance cardiovascular risk prediction in primary care subjects, given the simplicity of retrieving information on chest pain in combination with a single measurement of NT-proBNP for risk assessment in primary care. 


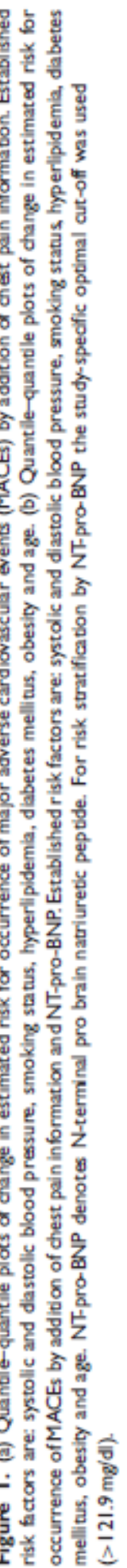




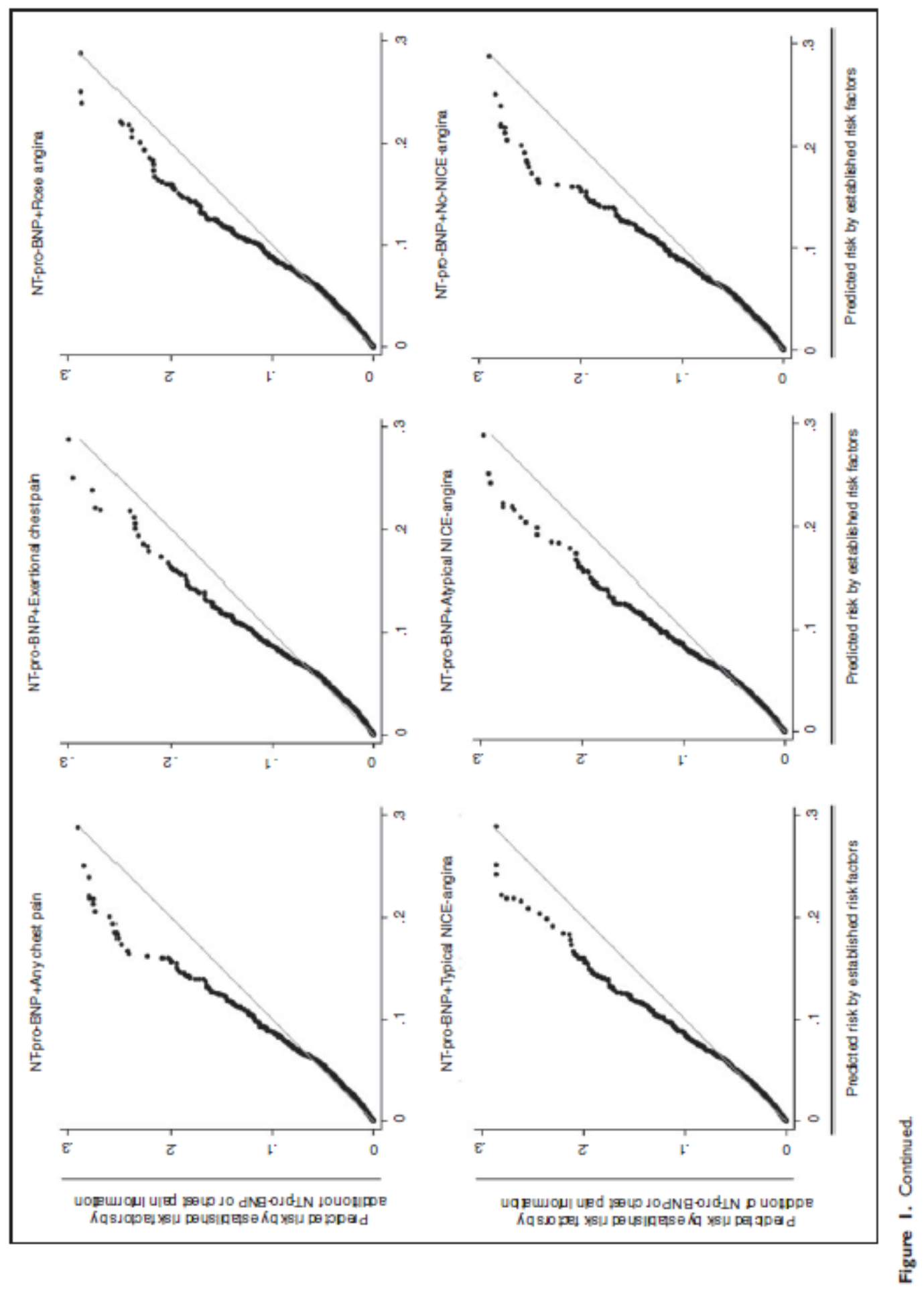




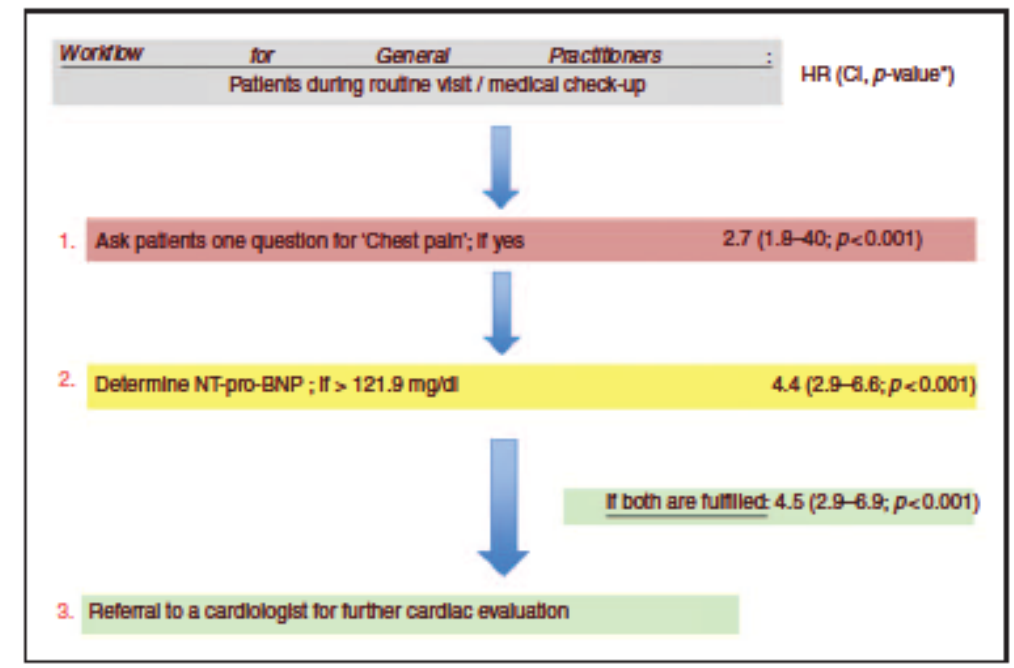

Figure 2. Algorithm for simple risk stratification in general practitioners office with risk stratification by asking one question for occurrence of chest pain and measuring NT-pro-BNP. NT-pro-BNP denotes N-terminal pro brain natriuretic peptide. For risk stratification by NT-pro-BNP the study-specific optimal cut-off was used (>121.9 mg/d); ( $\left(^{*}\right) \mathrm{HR}=\mathrm{Hazard}$ ratio for occurance of major cardiovascular event (MACE) within five years; $\mathrm{Cl}=$ Confidence interval.

Table I. Baseline characteristics and association with incident MACEs

\begin{tabular}{|c|c|c|c|c|}
\hline Characteristics & Total $\left.\right|^{2}$ & None-MACE ${ }^{2}$ & $\mathrm{MACE}^{2}$ & $\mathrm{HR}^{\prime}(95 \% \mathrm{Cl}) ;$ p-value \\
\hline Age, mean (SD). years ${ }^{5}$ & $55.6(13.8)$ & $55.4(13.7)$ & $68.1(10.5)$ & $1.08(1.06-1.10) ; p<0.001$ \\
\hline Female, $n(\%)$ & $3465(62.2)$ & $3429(62.8)$ & $36(33.0)$ & $3.40(2.28-5.07) ; p<0.001$ \\
\hline Hypertension, $n$ (\%) & $1900(34.1)$ & $1824(33.4)$ & $76(69.7)$ & $4.43(2.94-6.66) ; p<0.001$ \\
\hline Systolic blood pressure, mean(SD), $\mathrm{mmHg}^{6}$ & $131.5(18.1)$ & $131.4(18.2)$ & $139.8(16.0)$ & $1.02(1.02-1.03) ; p<0.001$ \\
\hline Diastolic blood pressure, mean(SD), $\mathrm{mmH}^{6}$ & $80.1(9.8)$ & $80.1(9.8)$ & $81.4(10.0)$ & $1.01(1.00-1.03) ; p<0.135$ \\
\hline Anthypertensive treatment, $n$ (\%) & $1660(31.5)$ & $1589(30.7)$ & $71(69.6)$ & $2.80(1.76-4.45) ; p<0.001$ \\
\hline $\mathrm{ACEl}$ or $\mathrm{ARB}, n(\%)$ & $1307(23.5)$ & $1247(22.8)$ & $60(50.1)$ & $3.99(2.74-5.83) ; p<0.001$ \\
\hline Beta-blocker, $n$ (\%) & $1068(20.2)$ & $1030(19.9)$ & $38(37.3)$ & $1.32(0.87-1.98) ; p<0.189$ \\
\hline Antagonist of calcium, $n$ ( $\%$ ) & $524(9.9)$ & $492(9.5)$ & $32(31.4)$ & $2.65(1.73-4.05) ; p<0.001$ \\
\hline Diuretics, $n(\%)$ & $654(12.4)$ & $631(12.2)$ & $23(22.6)$ & $1.24(0.77-1.98) ; p<0.375$ \\
\hline Diabetes mellitus, $n$ (\%) & $683(12.3)$ & $654(12.0)$ & $29(26.6)$ & $2.60(1.70-3.98): p<0.001$ \\
\hline Fasting plasma glucose, mean (SD), mg/di ${ }^{7}$ & $99.4(32.8)$ & $99.1(32.4)$ & $116.4(43.4)$ & $1.01(1.01-1.01) ; p<0.001$ \\
\hline HbAlc, mean (SD). \% & $5.5(0.8)$ & $5.5(0.8)$ & $5.9(0.9)$ & $1.50(1.36-1.66) ; p<0.001$ \\
\hline Oral drug treatment, $n$ (\%) & $436(8.3)$ & $415(8.0)$ & $21(20.6)$ & $1.87(1.15-3.04) ; p<0.011$ \\
\hline Insulin treatment, $n$ (\%) & $187(3.5)$ & $179(3.5)$ & $8(7.8)$ & $1.53(0.74-3.16) ; p<0.254$ \\
\hline Hyper lipidemia, $n$ (\%) & $1552(27.9)$ & $1501(27.5)$ & $51(46.8)$ & $2.26(1.55-3.29) ; p<0.001$ \\
\hline Statins, $n(\%)$ & $570(10.8)$ & $546(10.6)$ & $24(23.5)$ & $1.62(1.02-2.57) ; p<0.042$ \\
\hline Other lipid lowering drugs, $n$ (\%) & $154(2.9)$ & $147(2.8)$ & $7(6.9)$ & $1.64(0.76-3.53) ; p<0.209$ \\
\hline Total cholesterol, mean(SD), $\mathrm{mg} / \mathrm{dl}^{7}$ & $225.8(42.3)$ & $225.8(42.2)$ & $229.1(46.1)$ & $1.00(1.00-1.01): p<0.415$ \\
\hline HDL cholesterol, mean(SD), mg/di ${ }^{7}$ & $55.8(18.6)$ & $56.0(18.6)$ & $47.9(17.4)$ & $0.97(0.96-0.99) ; p<0.001$ \\
\hline $\mathrm{LDL}$ cholesterol, mean(SD), mg/di ${ }^{7}$ & $129.3(33.4)$ & $129.2(33.3)$ & $131.9(35.8)$ & $1.00(1.00-1.01) ; p<0.404$ \\
\hline Current smoker, $n$ ( $\%$ ) & $1097(21.3)$ & $1078(21.4)$ & $19(19.6)$ & $0.91(0.55-1.49) ; p<0.709$ \\
\hline Ex-smoker, $n(\%)$ & $1254(24.4)$ & $1224(24.3)$ & $30(30.9)$ & $1.28(0.84-1.95) ; p<0.252$ \\
\hline Family history of CAD, $n$ (\%) & $804(14.9)$ & $790(15.0)$ & $14(13.3)$ & $0.94(0.72-1.22) ; p<0.653$ \\
\hline Hip-to-waist ratio, mean (SD) & $1.13(0.13)$ & $1.13(0.13)$ & $1.07(0.10)$ & $0.04(0.01-0.12) ; p<0.001$ \\
\hline Body mass index, mean (SD), $\mathrm{kg}^{\prime} \mathrm{m}^{2}$ & $26.9(4.8)$ & $26.9(4.8)$ & $28.1(4.5)$ & $1.05(1.02-1.08) ; p<0.001$ \\
\hline Framingham Score-predicted risk, mean (SD) & $10.2(10.5)$ & $10.2(10.5)$ & $20.6(9.9)$ & $1.08(1.07-1.12) ; p<0.001$ \\
\hline \multicolumn{5}{|l|}{ Biomarkers } \\
\hline NT-pro-BNP ${ }^{3}$, median (interquartile range), pg/ml & $57.6(29.0-115.1)$ & $115.1(291.7)$ & $375.5(592.2)$ & $4.39(2.92-6.61): p<0.001$ \\
\hline Troponin $\mathrm{T}^{4}$, mean (SD). pg/dl & $0.01(0.01)$ & $0.01(0.00)$ & $0.02(0.04)$ & $1.22(1.17-1.28) ; p<0.001$ \\
\hline Creatinine mean (SD), mg/dl & $1.20(0.22)$ & $1.19(0.21)$ & $1.30(0.47)$ & $2.73(1.86-4.02) ; p<0.001$ \\
\hline
\end{tabular}

MACE, major cardiovascular event including denth from cardiovascular causes, non-fal myocardial irfarction or necessary revascularization by PCl or CABG; HR, hazard ratio; $\mathrm{C}$, confidence interval; SD, standard deviation, ACB, mgiotensin-converting errzyme inhbitor; ARB, angoters in receptor blocker, NT-pro-BNP, N-terminal pro-bran matriuretic peptide; CAD, coromary artery disease; 'Hazard ratio for increase of one unit if not otherwise stated. ${ }^{2}$ All percentages refer to number of subjects with existing data ${ }^{3} \mathrm{HR}$ for NT-pro BNP above $121.9 \mathrm{pg} / \mathrm{mL}$. ${ }^{4} \mathrm{HR}$ for an increase of $0.01 \mathrm{pg} / \mathrm{d}$. ${ }^{5} \mathrm{HR}$ for an increase of 10 years. ${ }^{4} \mathrm{HR}$ for an increase of $5 \mathrm{mmH}$. ${ }^{7} \mathrm{HR}$ for an increase of $10 \mathrm{mg} / \mathrm{d}$. 
Table 2. Hazard ratios for death by all causes, death by cardiovascular cause/non-fatal myocardial infarction and indident major cardiovascular events (MACE), according to definition of chest pain

\begin{tabular}{|c|c|c|c|c|c|c|}
\hline & \multicolumn{2}{|c|}{$\begin{array}{l}\text { Death by all causes } \\
(n=137)\end{array}$} & \multicolumn{2}{|c|}{$\begin{array}{l}\text { Death by cardiovascular } \\
\text { cause/non-fatal myocardial } \\
\text { infarction }(n=54)\end{array}$} & \multicolumn{2}{|l|}{$\operatorname{MACE}(n=109)$} \\
\hline & HR $(95 \% \mathrm{Cl})$ & $p$-value & HR $(95 \% \mathrm{Cl})$ & $p$-value & $\operatorname{HR}(95 \% \mathrm{Cl})$ & $p-$ value \\
\hline \multicolumn{7}{|c|}{ Any chest pain $(n=1463)$} \\
\hline Crude & $1.10(0.74-1.62)$ & 0.648 & $2.17(1.23-3.83)$ & 0.007 & $2.70(1.82-4.01)$ & $<0.001$ \\
\hline Adjusted (+) & $0.97(0.66-1.44)$ & 0.885 & $2.12(1.20-3.74)$ & 0.010 & $2.68(1.79-4.00)$ & $<0.001$ \\
\hline Adjusted $\left({ }^{++}\right)$ & $1.02(0.69-1.52)$ & 0.907 & $2.23(1.26-3.94)$ & 0.006 & $2.71(1.82-4.04)$ & $<0.001$ \\
\hline \multicolumn{7}{|c|}{ Exertional chest pain $(n=1003)$} \\
\hline Crude & $1.47(0.98-2.22)$ & 0.063 & $3.03(1.71-5.37)$ & $<0.001$ & $3.36(2.25-5.00)$ & $<0.001$ \\
\hline Adjusted (+) & $1.23(0.81-1.86)$ & 0.337 & $2.90(1.62-5.19)$ & $<0.001$ & $3.28(2.17-4.96)$ & $<0.001$ \\
\hline Adjusted $\left(^{++}\right)$ & $1.26(0.83-1.91)$ & 0.274 & $2.87(1.60-5.13)$ & $<0.001$ & $3.19(2.12-4.82)$ & $<0.001$ \\
\hline \multicolumn{7}{|c|}{ Rose angina ${ }^{15}(n=547)$} \\
\hline Crude & $1.29(0.76-2.18)$ & 0.344 & $2.82(1.46-5.43)$ & 0.002 & $2.78(1.75-4.41)$ & $<0.001$ \\
\hline Adjusted (†) & $1.14(0.66-1.95)$ & 0.645 & $2.96(1.51-5.82)$ & 0.002 & $2.97(1.82-4.84)$ & $<0.001$ \\
\hline Adjusted $\left({ }^{++}\right)$ & $1.16(0.68-1.99)$ & 0.589 & $2.93(1.50-5.76)$ & 0.002 & $2.95(1.81-4.82)$ & $<0.001$ \\
\hline \multicolumn{7}{|c|}{ NICE angina classification 17} \\
\hline \multicolumn{7}{|c|}{ Typical NICE angina $(n=1072)$} \\
\hline Crude & $1.19(0.79-1.78)$ & 0.408 & $2.50(1.44-4.34)$ & 0.001 & $3.15(2.15-4.61)$ & $<0.001$ \\
\hline Adjusted $\left(^{+}\right)$ & $1.07(0.71-1.61)$ & 0.746 & $2.44(1.40-4.26)$ & 0.002 & $3.18(2.16-4.69)$ & $<0.001$ \\
\hline Adjusted $\left({ }^{++}\right)$ & $1.11(0.74-1.66)$ & 0.620 & $2.40(1.38-4.16)$ & 0.002 & $3.14(2.13-4.63)$ & $<0.001$ \\
\hline \multicolumn{7}{|c|}{ Atypical NICE angina $(n=1115)$} \\
\hline Crude & $1.23(0.83-1.82)$ & 0.311 & $2.38(1.37-4.13)$ & 0.002 & $3.00(2.05-4.38)$ & $<0.001$ \\
\hline Adjusted $\left(^{+}\right)$ & $1.11(0.75-1.66)$ & 0.598 & $2.34(1.34-4.08)$ & 0.003 & $3.05(2.07-4.49)$ & $<0.001$ \\
\hline Adjusted $\left({ }^{++}\right)$ & $1.15(0.78-1.71)$ & 0.474 & $2.28(1.31-3.95)$ & 0.003 & $3.00(2.03-4.43)$ & $<0.001$ \\
\hline \multicolumn{7}{|c|}{ No NICE angina $(n=1463)$} \\
\hline Crude & $1.10(0.74-1.62)$ & 0.648 & $2.17(1.23-3.83)$ & 0.007 & $2.70(1.82-4.01)$ & $<0.001$ \\
\hline Adjusted $\left(^{+}\right)$ & $0.97(0.66-1.44)$ & 0.885 & $2.12(1.20-3.74)$ & 0.010 & $2.68(1.79-4.00)$ & $<0.001$ \\
\hline Adjusted $\left({ }^{++}\right)$ & $1.02(0.69-1.52)$ & 0.907 & $2.23(1.26-3.94)$ & 0.006 & $2.71(1.82-4.04)$ & $<0.001$ \\
\hline
\end{tabular}

HR, hazard ratio; $\mathrm{C}$, confidence interval; $\left(^{+}\right)=$Data were adjusted for the following variables: age at baseline (continuous) and gender (birary). $(++)=$ Data were adjusted for the following variables age at baseline (continuous), gender (bimary), smoleing staus (binary), hypertension (bimry). hyperlipidernia (binary), obesity (binary) and dabetes (birary). 
Table 3. C-statistics for Cox-regression models based on different definitions of chest pain and the addition of NT-pro-BNP for predicting incident major cardiovascular events (MACEs)

\begin{tabular}{|c|c|c|}
\hline & \multicolumn{2}{|l|}{ MACE } \\
\hline & C stutisties (") & prolue (†) \\
\hline \multicolumn{3}{|l|}{ Any chest pain } \\
\hline Established risk factors $\left({ }^{+}\right)$ & 0.774 & Reference model \\
\hline 'Any chest pain' & 0.632 & - \\
\hline NT-pro-BNP $\left({ }^{*}\right)$ & 0.672 & - \\
\hline Established risk factors $\left({ }^{*}\right)$ plus 'Any chest pain' & 0.806 & - \\
\hline Estimated difference with the addition of 'Any chest pain' & 0.025 & 0.024 \\
\hline Established risk factors $\left({ }^{*}\right)$ plus 'Any chest pain' and NT-pro-BNP $\left({ }^{* *}\right)$ & 0.824 & - \\
\hline Estimated difference with the addition of 'Any chest pain' and NT-pro-BNP (**) & 0.043 & 0.003 \\
\hline \multicolumn{3}{|l|}{ Exertional chest pain } \\
\hline Established risk factors $\left({ }^{+}\right)$ & 0.774 & Reference model \\
\hline 'Exertional chest pain' & 0.647 & - \\
\hline NT-pro-BNP $\left({ }^{\star *}\right)$ & 0.672 & - \\
\hline Established risk factors $\left({ }^{*}\right)$ plus 'Exertional chest pain' & 0.812 & - \\
\hline Estimated difference with the addition of 'Exertional chest pain' & 0.034 & 0.012 \\
\hline Established risk factors $\left({ }^{*}\right)$ plus 'Exertional chest pain' and NT-pro-BNP (**) & 0.830 & - \\
\hline Estimated difference with the addition of 'Exertional chest pain' and NT-pro-BNP ("*) & 0.048 & 0.003 \\
\hline \multicolumn{3}{|l|}{ Rose angina ${ }^{15}$} \\
\hline Established risk factors $\left({ }^{*}\right)$ & 0.774 & Reference model \\
\hline 'Rose angina' & 0.579 & - \\
\hline Nt-pro-BNP (**) & 0.672 & - \\
\hline Established risk factors $\left({ }^{*}\right)$ plus 'Rose angina' & 0.798 & - \\
\hline Estimated difference with the addition of 'Rose angina' & 0.020 & 0.054 \\
\hline Established risk factors $\left({ }^{*}\right)$ plus 'Rose angina' and NT-pro-BNP $\left({ }^{* *}\right)$ & 0.814 & - \\
\hline Estimated difference with the addition of 'Rose angina' and NT-pro-BNP (**) & 0.036 & 0.007 \\
\hline
\end{tabular}

NTpro-BNP, Neerminal pro-brain natriuretic peptide. (")= Etablished risk factors systolic blood pressure, dastolic blood pressure, smoking status, hyperlipidemia, diabetes mellitus, obesity and age $(\mathrm{N})=\mathrm{NT}$-pro-BNP $>121.9 \mathrm{mg} / \mathrm{d}$ ( = study-specific optimal cut-off derived from receiver operating characteristic malysis)). $\left({ }^{+}\right)=$Only determined for subjects with complete dita on established risk factors and NTpro-BNP measurement ( $\left.n=4734\right)$.

Table 4a. IDI of 5-year predicted risk for occurrence of major cardiovascular events (MACEs) by addition of chest pain information or NT-pro-BNP

\begin{tabular}{lll}
\hline & & $\begin{array}{l}\text { Relative change in higher risk } \\
\text { category for cases and lower risk } \\
\text { category in non-cases (\%) }\end{array}$ \\
\hline Any chest pain & $0.65(0.049)$ & 15.3 \\
Exer tional chest pain & $0.96(0.029)$ & 20.9 \\
Rose angina $^{15}$ & $0.68(0.049)$ & 15.7 \\
Typical NICE Angina $^{17}$ & $0.92(0.013)$ & 19.8 \\
Atypical NICA Angina $^{17}$ & $0.81(0.019)$ & 18.7 \\
No NICE Angina & $0.65(0.049)$ & 15.3 \\
NT-pro-BNP & $1.13(0.001)$ & 20.8 \\
\hline
\end{tabular}

NRL, net reclassfication improvement, NT-pro-BNP, N-terminal pro-brain natriuretic peptide, IDA, integrated discriminxion improvement (difference of averaged improvement in sensitivity and averaged increase in l-specificity); NT-pro-BNP, N-terminal pro-brain matriuretic peptide $>121.9$ mgldil (=studyspecific optimal cut-off derived from receiver opersing characteristic malysis). 
Table 4b. IDI of 5-year predicted risk for occurrence of MACEs by addition of chest pain information and NTpro-BNP

\begin{tabular}{lll}
\hline & & $\begin{array}{l}\text { Relative change in higher risk } \\
\text { category for cases and lower risk } \\
\text { category in non-cases (\%) }\end{array}$ \\
\hline Any chest pain + NT-pro-BNP & IDF $\%$ (p-value) & 40.7 \\
Exertional chest pain + NT-pro-BNP & $2.04(<0.001)$ & 48.5 \\
Rose angina ${ }^{15}+$ NT-pro-BNP & $2.47(<0.001)$ & 42.1 \\
Typical NICE Angina ${ }^{17}+$ NT-pro-BNP & $2.11(<0.001)$ & 42.1 \\
Atypical NICE Angina ${ }^{17}+$ NT-pro-BNP & $2.11(<0.001)$ & 36.5 \\
No NICE Angina ${ }^{17}+$ NT-pro-BNP & $1.99(<0.001)$ & 40.7 \\
\hline
\end{tabular}

NRL, net reclassification improvement, IDL, integated discriminaion improvement (dfference of averased improvement in sensitivity and averaged increase in 1-specificity); NT-pro-BNP. N-terminal pro-brain natriuretic peptide $>121.9 \mathrm{mg}$ dll (=study-ppecific optimal cut-off derived from receiver operaing chancteristic analysis); *Only determined for subjects with complete dita on established risk factors and NT-pro-BNP mensurement $(n=4734)$.

\section{Acknowledgement}

DETECT (Diabetes Cardiovascular Risk-Evaluation: Targets and Essential Data for Commitment of Treatment) is a cross-sectional and prospective-longitudinal, nationwide clinical epidemiological study.

Members of the DETECT-Study group include: Principal investigator: Professor Dr H-U Wittchen; Staff members: Dipl-Psych L Pieper, Dipl-Math J Klotsche, Dr T Eichler, Dr H Glaesmer, E Katze; Steering Committee: Professor Dr H Lehnert (Lübeck), Professor Dr GK Stalla (München), Professor Dr AM Zeiher (Frankfurt); Advisory Board: Professor Dr W März (Heidelberg/Graz), Professor Dr S Silber (München), Professor Dr U Koch (Hamburg), Priv- Doz Dr D Pittrow (München/Dresden), Professor Dr M Wehling (Mannheim), Dr D Leistner (Frankfurt), Dr HJ Schneider (München), Dr C Sievers (München).

\section{Funding}

DETECT is supported by an unrestricted educational grant of Pfizer GmbH, Karlsruhe, Germany.

\section{Conflict of interest}

None of the authors have any conflict of interest pertaining to the data presented, or have published or submitted any related papers from the same study.

\section{References}

1. Reddy KS. Cardiovascular disease in non-Western countries. N Engl J Med 2004; 350: $2438-2440$.

2. Heberden W. Commentaries on the History and Cure of Disease. London: TPayne, 1802. 3. Sekhri N, Feder GS, Junghans C, Hemingway H and Timmis AD. How effective are rapid access chest pain clinics? Prognosis of incident angina and non-cardiac chest pain in 8762 consecutive patients. Heart 2007; 93: 458-463.

4. Giugliano RP, Lloyd-Jones DM, Camargo Jr CA, Makary MA and O’Donnell CJ. Association of unstable angina guideline care with improved survival. Arch Intern Med 2000; 160: $1775-1780$. 
5. Braunwald E, Antman EM, Beasley JW, et al. ACC/AHA 2002 guideline update for the management of patients with unstable angina and non-ST-segment elevation myocardial infarction-summary article: a report of the American College of Cardiology/American Heart Association task force on practice guidelines (Committee on the Management of Patients With Unstable Angina). J Am Coll Cardiol 2002; 40: 1366-1374.

6. Bassand JP, Hamm CW, Ardissino D, et al. Guidelines for the diagnosis and treatment of non-ST-segment elevation acute coronary syndromes. Eur Heart J 2007; 28: 1598-1660. 7. Sorlie PD, Cooper L, Schreiner PJ, Rosamond W and Szklo M. Repeatability and validity of the Rose questionnaire for angina pectoris in the Atherosclerosis Risk in Communities Study. J Clin Epidemiol 1996; 49: 719-725.

8. LaCroix AZ, Guralnik JM, Curb JD, Wallace RB, Ostfeld AM and Hennekens CH. Chest pain and coronary heart disease mortality among older men and women in three communities. Circulation 1990; 81: 437-446.

9. Lampe FC, Whincup PH, Wannamethee SG, Ebrahim S, Walker M and Shaper AG. Chest pain on questionnaire and prediction of major ischaemic heart disease events in men. Eur Heart J 1998; 19: 63-73.

10. Erhardt L, Herlitz J, Bossaert L, et al. Task force on the management of chest pain. Eur Heart J 2002; 23: 1153-1176.

11. Bhopal RS, Bansal N, Fischbacher C, Brown H and Capewell S. Ethnic variations in chest pain and angina in men and women: Scottish Ethnicity and Health Linkage Study of 4.65 million people. Eur J Cardiovasc Prev Rehabil 2011; in press.

12. Zethelius B, Berglund L, Sundstrom J, et al. Use of multiple biomarkers to improve the prediction of death from cardiovascular causes. N Engl J Med 2008; 358: 2107-2116.

13. Wannamethee SG, Welsh P, Lowe GD, et al. N-terminal pro-brain natriuretic Peptide is a more useful predictor of cardiovascular disease risk than $\mathrm{C}$-reactive protein in older men with and without pre-existing cardiovascular disease. J Am Coll Cardiol 58: 56-64.

14. Wittchen HU, Glaesmer H, Marz W, et al. Cardiovascular risk factors in primary care:

methods and baseline prevalence rates - the DETECT program. Curr Med Res Opin 2005; 21: 619-630.

15. Rose GA. The diagnosis of ischaemic heart pain and intermittent claudication in field surveys. Bull World Health Organ 1962; 27: 645-658.

16. Lallukka T, Manderbacka K, Keskimaki I, et al. Angina pectoris: relation of

epidemiological survey to registry data. Eur J Cardiovasc Prev Rehabil 2011; 18: 621-626.

17. National Institute for Health and Clinical Excellence (NICE). Chest pain of recent onset:

Assessment and diagnosis of recent onset chest pain or discomfort of suspected cardiac origin.

Clinical Guidance CG95, National Institute for Health and Clinical Excellence, London, 2010, http://guidanceniceorguk/CG95.

18. James SK, Lindahl B, Siegbahn A, et al. N-terminal probrain natriuretic peptide and other risk markers for the separate prediction of mortality and subsequent myocardial infarction in patients with unstable coronary artery disease: a Global Utilization of Strategies To Open occluded arteries (GUSTO)-IV substudy. Circulation 2003; 108: 275-281.

19. Pearson TA, Blair SN, Daniels SR, et al. AHA Guidelines for Primary Prevention of Cardiovascular Disease and Stroke: 2002 Update: Consensus Panel Guide to Comprehensive Risk Reduction for Adult Patients Without Coronary or Other Atherosclerotic Vascular Diseases. American Heart Association Science Advisory and Coordinating Committee. Circulation 2002; 106: 388-391.

20. Third Report of the National Cholesterol Education Program (NCEP) Expert Panel on Detection, Evaluation, and Treatment of High Blood Cholesterol in Adults (Adult Treatment Panel III) final report. Circulation 2002; 106: 3143-3421. 
21. Simper D, Wang S, Deb A, et al. Endothelial progenitor cells are decreased in blood of cardiac allograft patients with vasculopathy and endothelial cells of noncardiac origin are enriched in transplant atherosclerosis. Circulation 2003; 108: 143-149.

22. Wilson PW, D'Agostino RB, Levy D, Belanger AM, Silbershatz H and Kannel WB. Prediction of coronary heart disease using risk factor categories. Circulation 1998; 97: 18371847.

23. Pencina $\mathrm{MJ}$ and D'Agostino RB. Overall $\mathrm{C}$ as a measure of discrimination in survival analysis: model specific population value and confidence interval estimation. Stat Med 2004; 23: 2109-2123.

24. Pencina MJ, D'Agostino Sr RB, D'Agostino Jr RB and Vasan RS. Evaluating the added predictive ability of a new marker: from area under the ROC curve to reclassification and beyond. Stat Med 2008; 27: 157-172; discussion 207-212.

25. Jones M, Rait G, Falconer J and Feder G. Systematic review: prognosis of angina in primary care. Fam Pract 2006; 23: 520-528.

26. Buckley BS, Simpson CR, McLernon DJ, Murphy AW and Hannaford PC. Five year prognosis in patients with angina identified in primary care: incident cohort study. BMJ 2009; 339: b3058.

27. Wang TJ, Gona P, Larson MG, et al. Multiple biomarkers for the prediction of first major cardiovascular events and death. N Engl J Med 2006; 355: 2631-2639. 28. de Lemos JA and Rohatgi A. Separating the contenders from the pretenders: competitive high-throughput biomarker screening in large population-based studies. Circulation 121: 2381-2383.

29. Olsen MH, Hansen TW, Christensen MK, et al. N-terminal pro-brain natriuretic peptide, but not high sensitivity C-reactive protein, improves cardiovascular risk prediction in the general population. Eur Heart J 2007; 28: 1374-1381.

30. Melander O, Newton-Cheh C, Almgren P, et al. Novel and conventional biomarkers for prediction of incident cardiovascular events in the community. JAMA 2009; 302: 49-57. 31. Blankenberg S, Zeller T, Saarela O, et al. Contribution of 30 biomarkers to 10-year cardiovascular risk estimation in 2 population cohorts: the MONICA, risk, genetics, archiving, and monograph (MORGAM) biomarker project. Circulation 121: 2388-2397. 